\title{
Age-by-disease biological interactions: implications for late-life depression
}

\author{
Brandon C. McKinney ${ }^{1}$, Hyunjung $\mathrm{Oh}^{1,2}$ and Etienne Sibille ${ }^{1,2 *}$ \\ ' Department of Psychiatry, University of Pittsburgh, Pittsburgh, PA, USA \\ ${ }^{2}$ Center for Neuroscience, University of Pittsburgh, Pittsburgh, PA, USA
}

Edited by:

Thomas Flatt, Vetmeduni Vienna,

Austria

Reviewed by:

Sangwon F. Kim, University of

Pennsylvania, USA

Haim Cohen, Bar-llan University, Israel

${ }^{*}$ Correspondence:

Etienne Sibille, Department of Psychiatry, University of Pittsburgh,

Bridgeside Point II, Suite 231,

Pittsburgh, PA 15219, USA

e-mail: sibilleel@upmc.edu
Onset of depressive symptoms after the age of 65, or late-life depression (LLD), is common and poses a significant burden on affected individuals, caretakers, and society. Evidence suggests a unique biological basis for LLD, but current hypotheses do not account for its pathophysiological complexity. Here we propose a novel etiological framework for LLD, the age-by-disease biological interaction hypothesis, based on the observations that the subset of genes that undergoes lifelong progressive changes in expression is restricted to a specific set of biological processes, and that a disproportionate number of these age-dependent genes have been previously and similarly implicated in neurodegenerative and neuropsychiatric disorders, including depression. The age-by-disease biological interaction hypothesis posits that age-dependent biological processes (i) are "pushed" in LLD-promoting directions by changes in gene expression naturally occurring during brain aging, which (ii) directly contribute to pathophysiological mechanisms of LLD, and (iii) that individual variability in rates of age-dependent changes determines risk or resiliency to develop age-related disorders, including LLD. We review observations supporting this hypothesis, including consistent and specific age-dependent changes in brain gene expression and their overlap with neuropsychiatric and neurodegenerative disease pathways. We then review preliminary reports supporting the genetic component of this hypothesis. Other potential biological mediators of age-dependent gene changes are proposed. We speculate that studies examining the relative contribution of these mechanisms to age-dependent changes and related disease mechanisms will not only provide critical information on the biology of normal aging of the human brain, but will inform our understanding of age-dependent diseases, in time fostering the development of new interventions for prevention and treatment of age-dependent diseases, including LLD.

Keywords: late-life depression, depression, molecular aging, gene expression, telomere, oxidative stress, epigenetic modifications

\section{INTRODUCTION}

Among elderly individuals, depressive symptoms are common and burdensome. Approximately $1 \%$ of individuals over the age of 65 meet criteria for major depressive disorder (MDD), as defined by the diagnostic and statistical manual of mental disorders, fourth edition, text revision (DSMIV-TR; American Psychiatric Association, 2000), a prevalence lower than that in younger adults (Kessler etal., 2003). Another 15-25\%, however, experience depressive symptoms that, while not meeting criteria for MDD, do cause significant distress and interfere with daily functioning (Koenig and Blazer, 1992). In this article, the term late-life depression (LLD) will be used to refer to individuals over the age of 65 who for the first time in their lives meet criteria for MDD or display clinically significant depressive symptoms. Individuals with LLD experience greater functional disability (Dombrovski et al., 2007) and cognitive decline (Lenze et al., 2005) than those without. Further, they are at increased risk of morbidity and mortality from medical illness (Ganguli et al., 2002). LLD also appears to contribute to increased rates of suicide among older individuals (Van Orden and Conwell, 2011).
The biological substrates of LLD are being characterized and several hypotheses for the etiology and pathophysiology of LLD have been proposed, including the vascular hypothesis (Alexopoulos et al., 1997), inflammation hypothesis (Alexopoulos and Morimoto, 2011), and dementia prodrome hypothesis (Byers and Yaffe, 2011; reviewed in McKinney and Sibille, 2012). Here, we propose an alternative and complementary hypothesis, which we termed the age-by-disease biological interaction hypothesis of LLD. Central to this hypothesis is the concept of molecular aging of the human brain. An earlier version of this hypothesis has been described elsewhere (McKinney and Sibille, 2012).

\section{MOLECULAR AGING OF THE HUMAN BRAIN}

Despite its critical importance to a population that is growing older, "normal" brain aging is understudied. This may be due to the often expressed, but false belief held by many that aging is inescapable, broad-ranging and non-specific. Studies that have investigated biological aging have revealed specific changes and thus avenues for intervention. At the cellular level in the human brain, morphological and stereological studies reveal a decrease 
in neuron volumes, a small loss or no change in cell numbers (Morrison and Hof, 1997; Pakkenberg and Gundersen, 1997), and a progressive thinning of cortical thickness, affecting both gray and white matter (Resnick et al., 2003; Sowell et al., 2003). Similar structural changes with age have been demonstrated in the brains of animal models (Jucker and Ingram, 1997; Peters, 2002). At the molecular level in animal models, less than $10 \%$ of brainexpressed genes exhibit age-related changes in gene expression (Lee et al., 1999, 2000; Jiang et al., 2001; Blalock et al., 2003; Sibille et al., 2007). Similar numbers have been reported in studies of human tissue (Lu et al., 2004; Avramopoulos et al., 2011). In one such study of human tissue from the prefrontal cortices of subjects aged 13-79, our group used gene microarray technology to investigate age-related changes in gene expression and reported that approximately $7.5 \%$ of genes changed significantly with aging (Erraji-Benchekroun et al., 2005). Other studies have confirmed these results, identifying a maximum of $\sim 10 \%$ of all detected genes, depending on sample size and analytical power of the respective studies (Yankner et al., 2008; Glorioso et al., 2011). Of note, not only is the identity of the genes and gene classes that are affected with aging consistent among studies, but so are the directions of change.

Interestingly, the identity of the genes whose expression changes with age suggests that specific cellular populations and biological processes are selectively affected by the aging process. For instance, expression of genes playing a role in glial-mediated inflammation, oxidative stress responses, mitochondrial function, synaptic function and plasticity, and calcium regulation and neuropeptide signaling have consistently been shown across multiple studies to be affected by aging, while numerous other neuronal and glial genes remain apparently unchanged (Yankner et al., 2008; Glorioso and Sibille, 2011). Overall, age-upregulated genes are mostly of glial origin and related to inflammation and cellular defenses, while downregulated genes display mostly neuron-enriched transcripts relating to cellular communication and signaling (Erraji-Benchekroun et al., 2005).

Using the expression levels of the age-dependent genes and their expected trajectories with age, we have generated predicted ages of individual subjects from which the brain tissue was sampled, and demonstrated that this predicted age is highly correlated with the chronological age of that individual (Erraji-Benchekroun et al., 2005; Glorioso and Sibille, 2011; Glorioso et al., 2011). We have termed this predicted age the "molecular age". These findings suggest that gene expression changes with age can be used as biomarkers for brain aging.

\section{MOLECULAR AGING AND BRAIN-RELATED DISEASE PATHWAYS}

This correlation between molecular and chronological ages is robust in individuals without neurodegenerative and neuropsychiatric disorders (Erraji-Benchekroun et al., 2005), but investigations of individual genes suggest that the molecular age or individual gene trajectory can deviate from chronological age in individuals with these disorders. To illustrate this phenomenon, one can look at somatostatin (SST), a signaling neuropeptide that is expressed in a subpopulation of gamma-aminobutyric acid (GABA)-positive inhibitory interneurons (Viollet et al., 2008). We have demonstrated that expression of SST decreases progressively with age in individuals without neurodegenerative and neuropsychiatric disorders such that expression levels at 70 years of age are approximately $40-50 \%$ of those at 20 years of age (ErrajiBenchekroun et al., 2005; Tripp et al., 2011; Figure 1A). SST is also downregulated in individuals with MDD. Interestingly, the magnitude and direction of change in SST expression with age is similar to that of control individuals, but the absolute values of SST expression are lower at most ages in individuals with MDD compared to those without (Sibille et al., 2011; Tripp et al., 2011; Figure 1A, top panel). Similar findings have been observed in subjects with schizophrenia (Morris et al., 2008; Figure 1A, bottom panel).

The relationship between gene expression of a disorderassociated gene and aging is not limited to SST. In fact, genomewide investigations have reported that up to a third of ageregulated genes in the human brain have been at some point associated in the literature with neurodegenerative (Alzheimer's, Parkinson's, and Huntington's diseases, amyotrophic lateral sclerosis) and neuropsychiatric disorders (bipolar depression, major depression, and schizophrenia; see Figure 3 in Glorioso et al., 2011 for details). Not only do the genes relevant to these brain disorders show age-related changes, but the direction of the changes that occur with age is almost always in the direction thought to cause or promote diseases. See Table 1 in Glorioso et al. (2011) for a list of approximately 40 top candidate disease genes that exhibit agreement between age- and disease-related changes. Studies of gene expression in Alzheimer's disease (AD) and normal aging find that most of the changes in gene expression that occur in normal aging also occur in AD, only in greater magnitude (Miller et al., 2008; Avramopoulos et al., 2011). The difference in the magnitude of change in gene expression between individuals with brain disease and those without during aging is attributable to the disease. Our hypothesis posits that it is these changes that drive the disease process; however, it cannot be ruled out that these additional changes in magnitude are the result of the disease process rather than the cause. That said, there are certainly genes for which it is known that the age-related direction of change in gene expression directly causes, rather than is a consequence of, the disease, for example, the Parkinson's disease genes $\mathrm{PINK}_{1}$ and $\mathrm{DJ}_{1} / \mathrm{PARK}_{7}$. The expression of these genes progressively declines with age and it is known that individuals with loss of expression/function mutations in these genes develop Parkinson's disease (Schapira, 2008). In contrast, relatively fewer of the larger pool of genes that do not display age-dependent changes $(<5 \%)$, are associated with neurodegenerative and neuropsychiatric diseases.

Recently, we have further investigated the relationship between age, gene changes, and neuropsychiatric disorders, specifically in the context of MDD (Douillard-Guilloux et al., 2012). Results demonstrated that most MDD-related genes were frequently ageregulated in both MDD and in control subjects, and that the effects of MDD and age were positively correlated. Moreover, most genes that are age-dependent in control subjects displayed greater age effects in MDD subjects, and the overall increased prevalence of age effects on genes in MDD subjects corresponded to similar trends in controls, rather than representing de novo age 


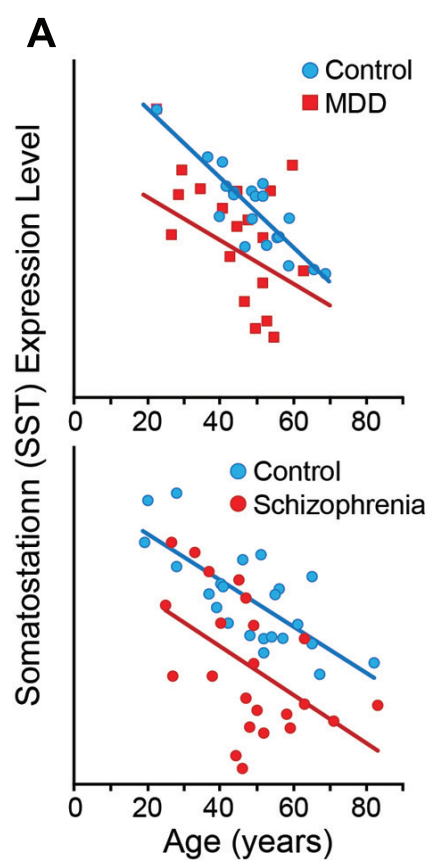

FIGURE 1 | (A) Somatostatin expression decreases with age in control subjects. Individuals with MDD (top panel) and schizophrenia (bottom panel) display lower levels of expression than control subjects at most ages, together prompting the hypothesis that decreased expression of somatostatin in depression and schizophrenia may represent early brain age-related molecular phenotypes in these individuals, which would render these subjects more vulnerable to developing psychiatric diseases. (B) Based on similar age and disease observations for numerous additional genes, we have proposed a model of "age-by-disease biological interaction" (Glorioso et al., 2011). In this model, change in expression of disease-related genes (a

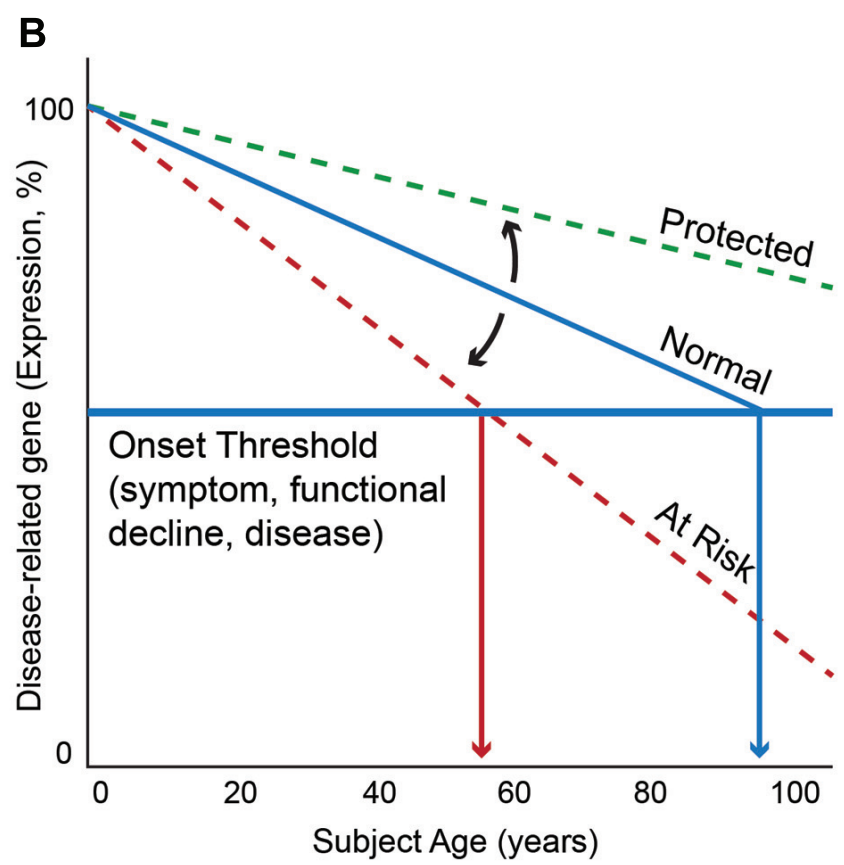

decrease is shown) across a threshold (horizontal blue line) marks the onset of disease symptoms. Changes in the trajectory of age-related changes in expression of disease-related genes ( $\mathrm{Y}$-axis) determine at what age (X-axis) or even if, an individual develops disease symptoms (vertical red arrows). Modulators (black arrows) may thus place an individual on "at risk" or "protected" trajectory. Per this model, individuals with LLD may have modulators, genetic or environmental, that place them on an "at risk" trajectory for developing mood symptoms. Figures are adapted from Morris etal. (2008), Tripp etal. (2011), Glorioso and Sibille (2011), and McKinney and Sibille (2012). effects. This systematic correlation between age-dependent and depression-related changes, with greater effects in depressed subjects, further suggests that normal brain aging is a risk factor for biological changes observed in MDD subjects.

One interpretation of these observations is that age-dependent changes (i.e., molecular aging) are on an earlier trajectory in individuals who develop MDD and potentially other neuropsychiatric disorders. However, it is important to note that these studies are cross-sectional and do not follow the longitudinal progression of gene changes within individuals, so it is not known whether age-dependent changes are on an earlier trajectory, or whether changes occurred at earlier time points and were fixed at lower levels, for instance in the case of SST. So while we hypothesize that age may be pushing the expression of genes in disordercausing directions, an alternate scenario is that of earlier and fixed changes, which then act as latent vulnerability factors that are revealed with advancing age, resulting in increased vulnerability to develop neurodegenerative and psychiatric disorders, including LLD.

\section{AGE-RELATED CHANGES IN GENE EXPRESSION APPEAR TO BE, IN PART, GENETICALLY MODULATED}

While molecular and chronological ages are highly correlated, we have also reported individual deviations from predicted ages
(Erraji-Benchekroun et al., 2005; Glorioso et al., 2011). The fact that molecular age can deviate from its chronological age suggests that modulating factors exist and may contribute to one's vulnerability to brain aging and to developing late-life brain disorders, such as LLD. In the age-by-disease biological interaction hypothesis we have proposed that those individuals with older predicted molecular ages compared to their chronological age may not only display greater biological brain aging, but may also be at greater risk of age-gated brain diseases, because gene expression of disease-related genes would have proceeded further in disease-promoting directions. Conversely, subjects with younger age-dependent gene trajectories and lower predicted molecular ages would be at lower risk, and may in fact display resiliency against LLD and other late-life disorders (Figure 1B). Environmental and genetic factors represent obvious candidates to modulate the trajectory of biological aging.

In a proof-of-principle study, our laboratory sought to demonstrate a genetic role in modulating the aging process. The above-described "molecular age" assay was used to characterize the brain tissue of individuals carrying different polymorphisms of the sirtuin genes (Glorioso et al., 2011), a family of genes previously demonstrated to modulate age and longevity in nematodes, insects, and rodents. We focused on SIRT5, due to prior report of altered expression for that gene in a rodent model of anticipated 
brain aging (Sibille etal., 2007). This study found that subjects carrying a low-expressing polymorphism of the SIRT5 gene had molecular ages that were older than actual chronological age, as measured in the three different areas of the cerebral cortex (i.e., BA9, BA24, and BA47) of human postmortem samples (Glorioso et al., 2011). Interestingly, this effect was not demonstrated in the amygdala, a brain area in which the low-expressing polymorphism of the SIRT5 gene does not appear to affect expression levels of SIRT5 as it does in the cerebral cortex. These findings at the molecular level are consistent with findings from studies of brain structure with age that show robust decreases in gray matter in the cerebral cortex but more variable decreases in the amygdala (Good et al., 2001). The effect of the low-expressing SIRT5 polymorphism on molecular age was accompanied by expression changes for a set of genes whose products are localized to the mitochondria, including PINK-1 and DJ-1, two Parkinson's disease-associated genes, in ways that would promote mitochondrial dysfunction-related diseases, including Parkinson's disease. This (correlative) proof-of-principle study suggests that factors that affect brain aging can potentially place an individual at higher risk for disease, through a mechanism by which it accelerates brain biological aging, and thus promotes changes in expression of disorder-relevant genes in disease-causing directions. With respect to Figure 1B, the low-expressing polymorphism of the SIRT5 gene can be thought of a modulator that puts one on the "at risk" trajectory. The converse of this model is that factors delaying age trajectories of gene changes may lead to younger brain molecular aging and potential resiliency toward developing functional declines and age-related disorders, including LLD (Figure 1B).

\section{PUTATIVE MECHANISMS FOR AGE-RELATED CHANGES IN GENE EXPRESSION}

The mechanisms by which age-related changes in gene expression occur are unknown. Candidate mediators include among others, loss of telomere integrity, increased oxidative stress, and epigenetic modifications.

\section{LOSS OF TELOMERE INTEGRITY}

Telomeres are regions of repetitive nucleotide sequences at each end of a chromosome. One of the hypothesized functions of telomeres is to deter the degradation of genes near the ends of chromosomes by instead allowing repetitive telomeres to shorten, a necessary part of chromosome replication. Telomeres are highly susceptible to oxidative stress because of their high content of guanines. As both chromosome replication and oxidative stress increase with age, one would expect telomeres to shorten with increased age. Indeed, in peripheral tissues, it has been consistently demonstrated that telomeres become shorter as one ages and once telomeres reach a critical length, irreversible arrest of cell division or apoptosis is triggered (Hayflick and Moorhead, 1961; Chin et al., 1999; Sharpless and DePinho, 2004; Flores and Blasco, 2009; Sahin and DePinho, 2012). Although telomere shortening has not yet been observed in the human brain, studies suggest that peripheral telomere length is a biomarker for aging. Leukocyte telomere shortening is positively correlated with the chronicity of stress and depression (Epel et al., 2004; Wolkowitz et al., 2011a) and is associated with incidence of age-related diseases such as myocardial infarction, stroke, and shorter lifespan (reviewed in Wolkowitz et al., 2011b). It is not known whether telomere shortening increases the risk of LLD, however, given the common pathway of aging and depression, the possibility cannot be excluded.

Recent animal studies suggest that the putative link between telomere integrity and depression-like behaviors extends to the brain, and that this link may be mediated by telomerase activity (Zhou et al., 2011). In that study, the expression of telomerase was decreased in the hippocampi of mice subjected to chronic mild stress, and hippocampal infusion of a telomerase inhibitor induced depressive-like behaviors that did not respond to antidepressant treatment (Zhou et al., 2011). Given that neurogenesis has been implicated in antidepressant responses in mice (Santarelli et al., 2003) and that the proliferation capacity of neural stem cells highly depend on telomerase activity (Ferron et al., 2009), the authors suggested telomerase activity may play a role in linking mechanisms of aging and depression (Zhou et al., 2011); although the translation of these observations to humans is contentious, due to very low rates of neurogenesis in adult human subjects (Bhardwaj et al., 2006).

The anti-apoptotic role of telomerase is thought to reflect its capacity of maintaining DNA integrity, however, recent studies have reported other putative functions (reviewed in Saretzki, 2009). Overexpression of telomerase reverse transcriptase (TERT), protects mouse neurons from excitotoxicity by improving basal mitochondrial membrane potential and $\mathrm{Ca}^{2+}$ uptake into mitochondria (Kang et al., 2004) and decreases cellular reactive oxygen species (ROS; Ahmed et al., 2008). Furthermore, TERT mediates the neurotrophic action of BDNF (Fu et al., 2002) and affects the global pattern of gene expression in the direction of cell survival, including upregulation of growth promoting genes (FGF5, EGFR, and etc.) and downregulation of cell-growth inhibitors such as IGFBPs (Smith et al., 2003; Choi et al., 2008). Changes in telomerase activity with age and MDD have yet to be explored in the human brain. However, given the fact that neurotrophic growth signaling, including reduced BDNF, is decreased in MDD (Sen et al., 2008; Guilloux et al., 2011; Tripp et al., 2011), we cannot exclude the possibility that telomeres and telomerase activity may significantly contribute to the mediation of stress and brain aging.

\section{INCREASED OXIDATIVE STRESS}

Oxidative stress is the damage caused to cells as a result of an imbalance between the production of ROS and the ability of the cells to reduce the ROS or repair the resulting damage. The degree of oxidative stress to cellular components, including DNA, correlates positively with age (Joseph et al., 2005; Epel, 2009; Wolkowitz et al., 2011b). Because of their high demand for energy and postmitotic status, neurons appear to be particularly sensitive to oxidative stress and thus aging.

One way in which oxidative stress may contribute to age-related changes in gene expression is via its direct effect on DNA. Lu etal. (2004) showed that age-related decrease in gene expression is related to the accumulation of oxidative DNA damage. The underlying mechanism was suggested that promoter regions 
with high GC contents are specifically vulnerable to oxidative damage. Oxidated promoter regions may potentially adopt different conformation and lose affinity for transcription factors (Lu etal., 2004). Damage on mitochondrial DNA (mtDNA), which is considered extremely vulnerable to oxidation due to its proximity to the site of ROS production, respiratory chain, and the absence of protective histone (Lee and Wei, 2007), results in downregulation of genes related to respiratory chain and further, energy metabolism impairment (Lin etal., 2002). Several studies showed that psychiatric diseases including MDD, bipolar disorder, and schizophrenia are associated with mitochondrial dysfunction (Rezin et al., 2009) and that accumulation of mtDNA damage induces mood disorderlike phenotypes as well as premature aging in mice (Trifunovic et al., 2004; Kasahara et al., 2006). These results support the idea that oxidative stress plays a role as a link between aging and depression.

Another direct way in which oxidative stress may contribute to age-related changes in gene expression is via its effect on transcription factors. For example, ROS are able to activate nuclear factor kappa B (NF- $\kappa \mathrm{B})$ by decreasing binding affinity of the inhibitory subunit, I $\kappa-\mathrm{B}$, to NF- $\kappa \mathrm{B}$, an observation made relevant by the fact that NF- $\kappa \mathrm{B}$ activity has been demonstrated to increase with aging and depression (Toliver-Kinsky et al., 1997; Koo et al., 2010). Also, transcription factors containing the zincfinger DNA binding motif appear to be especially susceptible to damage from oxidative stress due to their high cysteine residue content. As intracellular ROS accumulate, oxidation of the thiol residues in cysteine occurs and binding affinity for DNA is lost. One of the zinc-finger transcription factors, Sp1, an ubiquitous transcription factor for housekeeping genes and enzymes involved in glucose metabolism and DNA synthesis, has been demonstrated to have decreased DNA binding affinity with advancing age (Ammendola et al., 1992, 1994; Wu et al., 1996). Interestingly, genes with lower affinity binding sites to $\mathrm{Sp} 1$ are more influenced by oxidative stress than those containing high-affinity sites (Ammendola etal., 1992, 1994; Wu et al., 1996). Furthermore, ROS decreases telomerase activity. Antioxidant treatment normalizes catalytic activity of TERT and delays cellular senescence (Haendeler et al., 2004).

In addition to the conformational change of gene and transcription factors, ROS can act on various cellular signaling pathways to control gene expression. For example, ROS increase p53 signaling, which has been implicated in various neurodegenerative diseases and thought to mediate its effect by increasing expression of genes related to cell cycle arrest, DNA repair, and apoptosis in response to cellular stressors such as DNA damage and hypoxia (Lundberg et al., 2000). Another example is illustrated by p38 MAP Kinase (MAPK). When p38 MAPK is activated by oxidative stress, it promotes lamin B1 accumulation and expression of several transcription factors related to cellular senescence and apoptosis such as p53, CREB, C/EBP $\beta$, and ATF2 (Wagner and Nebreda, 2009; Barascu et al., 2012). Interestingly, selective p38 MAPK deletion in serotonergic neurons produces stress resilience in an animal model of depression by inhibiting serotonin transporter translocation to plasma membrane (Bruchas et al., 2011).
In summary, the main effect of oxidative stress on aging has been thought to be the accumulation of toxic, inactive molecules produced randomly by ROS. However, oxidative stress may also have an active role in aging and related diseases, through direct modification of DNA and transcription factor integrity and through indirect pathways regulating upstream modulators. These observations suggest that antioxidants may contribute to preventing biological changes and/or associated symptoms of depression, in addition to their potential anti-aging effects.

\section{EPIGENETIC MODIFICATIONS}

Epigenetic modifications, including DNA methylation and histone modification, regulate gene expression without changing the primary DNA sequence. Though classically viewed as a permanent event, recent data indicates that such modifications are influenced by genetic and environmental factors in adult organisms, including changes in methylation patterns across the lifespan (Numata et al., 2012). At the genome level, DNA methylation decreases with age. In contrast, $\mathrm{CpG}$ islands of many specific promoter regions that are typically not methylated become methylated with aging, including in promoters of tumor suppressor genes, estrogen receptor (ER), and insulin-like growth factor 2 (IGF2; Issa et al., 1994, 1996; So et al., 2006; Numata et al., 2012). Changes in DNA methylation at the glucocorticoid receptor, potentially due to early-life stress, was also correlated with altered vulnerability to psychiatric disorders and death by suicide in adults (McGowan et al., 2009).

Similarly, histone modifications such as acetylation, phosphorylation, symoylation, and methylation change with age. It was recently demonstrated that the aging-related deficit of longterm synaptic plasticity in the rodent hippocampus is related to decreased BDNF expression secondary to decreased acetylation of histones residing at the BDNF promoter region (Zeng et al., 2011). This observation fits well with human studies demonstrating reduced BDNF function in aging and depression (Webster et al., 2002; Guilloux et al., 2011). Also, changes in histone modifications in rodent systems may be protective against the effects of stress (Covington et al., 2011; Uchida et al., 2011). In support of a specific role for histone acetylation in age-related changes in gene expression, sirtuins (NAD-dependent histone deacetylases) have been implicated in longevity in yeast and invertebrates (Longo and Kennedy, 2006). A recent rodent study directly links SIRT1 to the risk of anxiety-like behaviors through its activity on monoamine oxidase $\mathrm{A}$ and serotonin levels (Libert etal., 2011). In the same study, the authors reported association between a single nucleotide polymorphism in the human SIRT1 gene and the risk of various psychiatric disorders such as anxiety disorder, panic disorder, and major depression. Furthermore, overexpression of SIRT6 in mice has been reported to increase lifespan and protect from diet-induced physiological damage (Kanfi et al., 2010, 2012) and SIRT6 knockout mice exhibit accelerated aging. Interestingly, decreased expression of SIRT6 has been observed during MDD (Abe et al., 2011). How SIRT6 mediates its effect on aging or is involved in MDD is not clear, but its functions as an HDAC (Michishita et al., 2008; Kawahara etal., 2009; Tennen et al., 2011) and in DNA repair (Mao etal., 2011) suggest that it may contribute to protecting 
against aging and psychiatric illness by maintaining telomere integrity or protecting against and repairing the effects of oxidative stress.

Together, the occurrence of epigenetic modifications during aging and in the context of neuropsychiatric disorders may thus provide mechanistic underpinnings for the proposed age-bydisease biological interaction hypothesis, through the dual role of longevity and other age-associated genes.

\section{SUMMARY AND IMPLICATIONS}

We propose a novel framework for investigating the development of late-life brain disorders, including LLD, which we term the ageby-disease biological interaction hypothesis. This paper expands upon an earlier version described elsewhere (McKinney and Sibille, 2012). This hypothesis posits that symptoms of LLD and other late-life brain disorders are the emerging properties of underlying biological changes, which in turn are supported by normal changes in the expression of multiple genes with age, including disease-related genes changing in disease-causing directions. Here, in addition to presenting the gene expression data on which the hypothesis is based, we discussed molecular mechanisms that may account for age-dependent gene expression changes, including loss of telomere integrity, increased oxidative stress, and epigenetic modifications. Importantly, this hypothesis complements existing hypotheses, including the vascular, inflammatory, and dementia prodrome hypotheses of LLD, but it differs in that it positions age-dependent gene expression changes as the mechanism potentially driving dysfunctions in multiple biological pathways, including vascular, inflammatory, and neurotrophic functions. A potential sequence of events is summarized in Figure 2. The purpose of this paper was to discuss the general framework. Examples of gene changes at the intersection of depression and aging were provided (e.g., SST and BDNF), but the exact nature and complexity of changes in multiple genes and pathways and their relevance to disease pathways will be described in details elsewhere.

The implications of this hypothesis for the prevention and treatment of LLD and other late-life brain disorders are exciting. Understanding the mechanisms mediating age-related changes in gene expression is expected to provide insight into pathophysiological mechanisms and potential targets for intervention into these disorders. Identifying key upstream hub genes mediating patterns of altered age-dependent changes would provide novel targets for further investigations. Although sirtuins and BDNF may represent obvious candidates, the large set of age-dependent genes ( $~ 10 \%$ of all genes; Erraji-Benchekroun et al., 2005) and its overlap with genes previously implicated in brain disorders (Glorioso et al., 2011) should be viewed as an enriched pool of candidate genes. Targeting such upstream factors (transcription or function) should represent productive research avenues. Early candidate interventions may include known interventions such as antidepressant medications, psychotherapy, exercise, and others. Investigating how these known interventions affect age-dependent changes in the function of critical genes may help in optimizing their implementation with respect to timing and duration of intervention for age-dependent disorders. Further, identifying genetic and environmental factors that slow or accelerate age-related

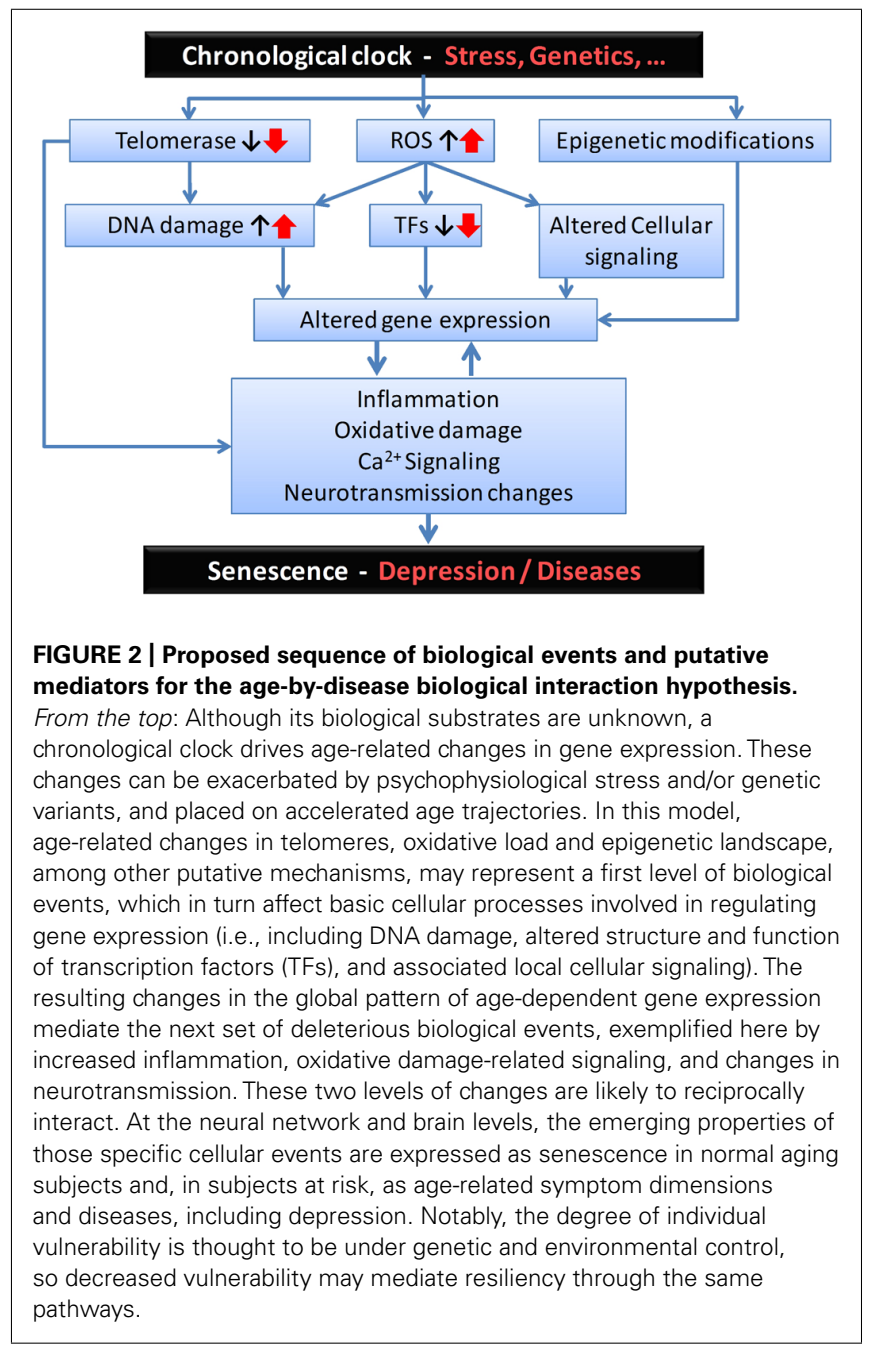

changes in gene function may lead to individualized strategies aimed at promoting resilience and successful aging. Other entry points and targets for intervention are likely to arise out of understanding the mechanisms by which gene expression changes with age, including determining the role of telomere integrity, oxidative stress, and epigenetic modifications. Finally, for the broader fields of aging and gerontology, the implication of this hypothesis is that it brings together research on normal aging more closely with the investigation of neuropsychiatric and neurodegenerative diseases. Indeed, our data firmly support the assertion that they may in fact be related facets of similar biological processes, and also provide the basis for a putative mechanism of age-by-disease biological interactions.

\section{ACKNOWLEDGMENTS}

This work was supported by the National Institute of Mental Health (NIMH) MH084060 and MH093723 grants to Etienne Sibille. The funding agency had no role in the study design, data collection and analysis, decision to publish, and preparation of manuscript. The content is solely the responsibility of the authors and does not necessarily represent the official views of the NIMH or the National Institutes of Health. 


\section{REFERENCES}

Abe, N., Uchida, S., Otsuki, K., Hobara, T., Yamagata, H., Higuchi, F., et al. (2011). Altered sirtuin deacetylase gene expression in patients with a mood disorder. J. Psychiatr. Res. 45, 1106-1112.

Ahmed, S., Passos, J. F., Birket, M. J., Beckmann, T., Brings, S., Peters, H., et al. (2008). Telomerase does not counteract telomere shortening but protects mitochondrial function under oxidative stress. J. Cell Sci. 121, 1046-1053.

Alexopoulos, G. S., Meyers, B. S., Young, R. C., Campbell, S., Silbersweig, D., and Charlson, M. (1997). 'Vascular depression' hypothesis. Arch. Gen. Psychiatry 54, 915-922.

Alexopoulos, G. S., and Morimoto, S. S. (2011). The inflammation hypothesis in geriatric depression. Int. J. Geriatr. Psychiatry doi: 10.1002/gps.2672 [Epub ahead of print].

American Psychiatric Association. (2000). Diagnostic Criteria from $D S M-I V-T R$. Washington, DC: American Psychiatric Association.

Ammendola, R., Mesuraca, M., Russo, T., and Cimino, F. (1992). Sp1 DNA binding efficiency is highly reduced in nuclear extracts from aged rat tissues. J. Biol. Chem. 267, 17944-17948.

Ammendola, R., Mesuraca, M., Russo, T., and Cimino, F. (1994). The DNAbinding efficiency of Spl is affected by redox changes. Eur. J. Biochem. 225, 483-489.

Avramopoulos, D., Szymanski, M., Wang, R., and Bassett, S. (2011). Gene expression reveals overlap between normal aging and Alzheimer's disease genes. Neurobiol. Aging 32, 2319.e272319.e34.

Barascu, A., Le Chalony, C., Pennarun, G., Genet, D., Imam, N., Lopez, B., etal. (2012). Oxidative stress induces an ATM-independent senescence pathway through p38 MAPKmediated lamin B1 accumulation. EMBO J. 31, 1080-1094.

Bhardwaj, R. D., Curtis, M. A., Spalding, K. L., Buchholz, B. A., Fink, D., BjorkEriksson, T., et al. (2006). Neocortical neurogenesis in humans is restricted to development. Proc. Natl. Acad. Sci. U.S.A. 103, 12564-12568.

Blalock, E. M., Chen, K. C., Sharrow, K., Herman, J. P., Porter, N. M., Foster, T. C., et al. (2003). Gene microarrays in hippocampal aging: statistical profiling identifies novel processes correlated with cognitive impairment. $J$. Neurosci. 23, 3807-3819.

Bruchas, M. R., Schindler, A. G., Shankar, H., Messinger, D. I., Miyatake, M., Land, B. B., et al. (2011). Selective p38alpha MAPK deletion in serotonergic neurons produces stress resilience in models of depression and addiction. Neuron 71, 498-511.

Byers, A. L., and Yaffe, K. (2011). Depression and risk of developing dementia. Nat. Rev. Neurol. 7 323-331.

Chin, L., Artandi, S. E., Shen, Q., Tam, A., Lee, S. L., Gottlieb, G. J., et al. (1999). p53 deficiency rescues the adverse effects of telomere loss and cooperates with telomere dysfunction to accelerate carcinogenesis. Cell 97, 527-538.

Choi, J., Southworth, L. K., Sarin, K. Y., Venteicher, A. S., Ma, W., Chang, W., et al. (2008). TERT promotes epithelial proliferation through transcriptional control of a Myc- and Wnt-related developmental program. PLoS Genet. 4, e10. doi: 10.1371/journal.pgen.0040010

Covington, H. E. III, Maze, I., Sun, H., Bomze, H. M., Demaio, K. D., Wu, E. Y., et al. (2011). A role for repressive histone methylation in cocaineinduced vulnerability to stress. Neuron 71, 656-670.

Dombrovski, A. Y., Mulsant, B. H., Houck, P. R., Mazumdar, S., Lenze, E. J., Andreescu, C., et al. (2007). Residual symptoms and recurrence during maintenance treatment of late-life depression. J. Affect. Disord. 103, $77-82$.

Douillard-Guilloux, G., Guilloux, J. P., Lewis, D. A., and Sibille, E. (2012). Anticipated brain molecular aging in major depression. Am. J. Geriatr. Psychiatry doi: 10.1097/JGP. 0b013e318266b7ad [Epub ahead of print].

Epel, E. S. (2009). Psychological and metabolic stress: a recipe for accelerated cellular aging? Hormones (Athens) 8, 7-22.

Epel, E. S., Blackburn, E. H., Lin, J., Dhabhar, F. S., Adler, N. E., Morrow, J. D., et al. (2004). Accelerated telomere shortening in response to life stress. Proc. Natl. Acad. Sci. U.S.A. 101, 17312-17315.

Erraji-Benchekroun, L., Underwood, M. D., Arango, V., Galfalvy, H., Pavlidis, P., Smyrniotopoulos, P., et al. (2005). Molecular aging in human prefrontal cortex is selective and continuous throughout adult life. Biol. Psychiatry 57, 549-558.

Ferron, S. R., Marques-Torrejon, M. A., Mira, H., Flores, I., Taylor, K., Blasco, M. A., et al. (2009). Telomere shortening in neural stem cells disrupts neuronal differentiation and neuritogenesis. J. Neurosci. 29, 14394-14407.

Flores, I., and Blasco, M. A. (2009). A p53-dependent response limits epidermal stem cell functionality and organismal size in mice with short telomeres. PLoS ONE 4, e4934. doi: 10.1371/journal.pone.0004934

Fu, W., Lu, C., and Mattson, M. P. (2002). Telomerase mediates the cell survival-promoting actions of brain-derived neurotrophic factor and secreted amyloid precursor protein in developing hip pocampal neurons. J. Neurosci. 22, 10710-10719.

Ganguli, M., Dodge, H. H., and Mulsant, B. H. (2002). Rates and predictors of mortality in an aging, rural, community-based cohort: the role of depression. Arch. Gen. Psychiatry 59, 1046-1052.

Glorioso, C., Oh, S., Douillard, G. G., and Sibille, E. (2011). Brain molecular aging, promotion of neurological disease and modulation by sirtuin 5 longevity gene polymorphism. Neu robiol. Dis. 41, 279-290.

Glorioso, C., and Sibille, E. (2011) Between destiny and disease: genetics and molecular pathways of human central nervous system aging. Prog. Neurobiol. 93, 165-181.

Good, C. D., Johnsrude, I. S., Ashburner J., Henson, R. N., Friston, K. J., and Frackowiak, R. S. (2001). A voxelbased morphometric study of ageing in 465 normal adult human brains. Neuroimage 14, 21-36.

Guilloux, J. P., Douillard-Guilloux, G., Kota, R., Wang, X., Gardier, A. M., Martinowich, K., et al. (2011). Molecular evidence for BDNF- and GABA-related dysfunctions in the amygdala of female subjects with major depression. Mol. Psychiatry doi: 10.1038/mp.2011.113 [Epub ahead of print].

Haendeler, J., Hoffmann, J., Diehl, J. F., Vasa, M., Spyridopoulos, I. Zeiher, A. M., et al. (2004). Antioxidants inhibit nuclear export of telomerase reverse transcriptase and delay replicative senescence of endothelial cells. Circ. Res. 94, 768-775.

Hayflick, L., and Moorhead, P. S. (1961). The serial cultivation of human diploid cell strains. Exp. Cell Res. 25, 585-621.

Issa, J. P., Ottaviano, Y. L., Celano, P., Hamilton, S. R., Davidson, N. E. and Baylin, S. B. (1994). Methylation of the oestrogen receptor $\mathrm{CpG}$ island links ageing and neoplasia in human colon. Nat. Genet. 7, 536-540.

Issa, J. P., Vertino, P. M., Boehm, C. D., Newsham, I. F., and Baylin, S B. (1996). Switch from monoallelic to biallelic human IGF2 promoter methylation during aging and carcinogenesis. Proc. Natl. Acad. Sci. U.S.A. 93, 11757-11762.
Jiang, C. H., Tsien, J. Z., Schultz, P. G., and $\mathrm{Hu}, \mathrm{Y}$. (2001). The effects of aging on gene expression in the hypothalamus and cortex of mice. Proc. Natl. Acad. Sci. U.S.A. 98, 1930-1934.

Joseph, J. A., Shukitt-Hale, B., Casadesus, G., and Fisher, D. (2005). Oxidative stress and inflammation in brain aging: nutritional considerations. Neurochem. Res. 30, 927-935.

Jucker, M., and Ingram, D. K. (1997). Murine models of brain aging and age-related neurodegenerative diseases. Behav. Brain Res. 85, 1-26.

Kanfi, Y., Naiman, S., Amir, G., Peshti, V., Zinman, G., Nahum, L., et al. (2012). The sirtuin SIRT6 regulates lifespan in male mice. Nature 483, 218-221.

Kanfi, Y., Peshti, V., Gil, R., Naiman, S., Nahum, L., Levin, E., et al. (2010). SIRT6 protects against pathological damage caused by diet-induced obesity. Aging Cell 9, 162-173.

Kang, H. J., Choi, Y. S., Hong, S. B., Kim, K. W., Woo, R. S., Won, S. J., et al. (2004). Ectopic expression of the catalytic subunit of telomerase protects against brain injury resulting from ischemia and NMDAinduced neurotoxicity. J. Neurosci. 24, 1280-1287.

Kasahara, T., Kubota, M., Miyauchi, T., Noda, Y., Mouri, A., Nabeshima, T., et al. (2006). Mice with neuronspecific accumulation of mitochondrial DNA mutations show mood disorder-like phenotypes. Mol. Psychiatry 11, 577-593.

Kawahara, T. L., Michishita, E., Adler, A. S., Damian, M., Berber, E., Lin, M., etal. (2009). SIRT6 links histone $\mathrm{H} 3$ lysine 9 deacetylation to NF-kappaB-dependent gene expression and organismal life span. Cell 136, 62-74.

Kessler, R. C., Berglund, P., Demler, O., Jin, R., Koretz, D., Merikangas, K. R., et al. (2003). The epidemiology of major depressive disorder: results from the National Comorbidity Survey Replication (NCS-R). JAMA 289, 3095-3105.

Koenig, H. G., and Blazer, D. G. (1992). Epidemiology of geriatric affective disorders. Clin. Geriatr. Med. 8, 235-251.

Koo, J. W., Russo, S. J., Ferguson, D., Nestler, E. J., and Duman, R. S. (2010). Nuclear factor-kappaB is a critical mediator of stress-impaired neurogenesis and depressive behavior. Proc. Natl. Acad. Sci. U.S.A. 107, 2669-2674

Lee, C. K., Klopp, R. G., Weindruch, R., and Prolla, T. A. (1999). Gene 
expression profile of aging and its retardation by caloric restriction. Science 285, 1390-1393.

Lee, C. K., Weindruch, R., and Prolla, T. A. (2000). Gene-expression profile of the ageing brain in mice. Nat. Genet. 25, 294-297.

Lee, H. C., and Wei, Y. H. (2007). Oxidative stress, mitochondrial DNA mutation, and apoptosis in aging. Exp. Biol. Med. (Maywood) 232, 592-606.

Lenze, E. J., Schulz, R., Martire, L. M., Zdaniuk, B., Glass, T., Kop, W. J., et al. (2005). The course of functional decline in older people with persistently elevated depressive symptoms: longitudinal findings from the Cardiovascular Health Study. J. Am. Geriatr. Soc. 53, 569-575.

Libert, S., Pointer, K., Bell, E. L., Das, A., Cohen, D. E., Asara, J. M., et al. (2011). SIRT1 activates MAO-A in the brain to mediate anxiety and exploratory drive. Cell 147, 1459-1472.

Lin, M. T., Simon, D. K., Ahn, C. H., Kim, L. M., and Beal, M. F. (2002). High aggregate burden of somatic mtDNA point mutations in aging and Alzheimer's disease brain. Hum. Mol. Genet. 11, 133-145.

Longo, V. D., and Kennedy, B. K. (2006). Sirtuins in aging and age-related disease. Cell 126, 257-268.

Lu, T., Pan, Y., Kao, S. Y., Li, C. Kohane, I., Chan, J., et al. (2004). Gene regulation and DNA damage in the ageing human brain. Nature 429 , 883-891.

Lundberg, A. S., Hahn, W. C., Gupta, P., and Weinberg, R. A. (2000). Genes involved in senescence and immortalization. Curr. Opin. Cell Biol. 12, 705-709.

Mao, Z., Hine, C., Tian, X., Van Meter, M., Au, M., Vaidya, A., et al. (2011). SIRT6 promotes DNA repair under stress by activating PARP1. Science 332, 1443-1446.

McGowan, P. O., Sasaki, A., D’Alessio, A. C., Dymov, S., Labonte, B., Szyf, M., et al. (2009). Epigenetic regulation of the glucocorticoid receptor in human brain associates with childhood abuse. Nat. Neurosci. 12, 342-348.

McKinney, B. C., and Sibille, E. (2012). The age-by-disease interaction hypothesis of late-life depression. Am. J. Geriatr. Psychiatry doi: $10.1097 /$ JGP.0b013e31826ce80d [Epub ahead of print]

Michishita, E., Mccord, R. A., Berber, E., Kioi, M., Padilla-Nash, H., Damian, M., et al. (2008). SIRT6 is a histone H3 lysine 9 deacetylase that modulates telomeric chromatin. Nature 452, 492-496.

Miller, J. A., Oldham, M. C., and Geschwind, D. H. (2008). A systems level analysis of transcriptional changes in Alzheimer's disease and normal aging. J. Neurosci. 28 1410-1420.

Morris, H. M., Hashimoto, T., and Lewis, D. A. (2008). Alterations in somatostatin mRNA expression in the dorsolateral prefrontal cortex of subjects with schizophrenia or schizoaffective disorder. Cereb. Cortex 18, 1575-1587.

Morrison, J. H., and Hof, P. R. (1997). Life and death of neurons in the aging brain. Science 278, 412-419.

Numata, S., Ye, T., Hyde, T. M., GuitartNavarro, X., Tao, R., Wininger, M. et al. (2012). DNA methylation signatures in development and aging of the human prefrontal cortex. Am. J. Hum. Genet. 90, 260-272.

Pakkenberg, B., and Gundersen, H. J. (1997). Neocortical neuron number in humans: effect of sex and age. $J$. Comp. Neurol. 384, 312-320.

Peters, A. (2002). Structural changes in the normally aging cerebral cortex of primates. Prog. Brain Res. 136, 455-465.

Resnick, S. M., Pham, D. L., Kraut, M. A., Zonderman, A. B., and Davatzikos, C. (2003). Longitudinal magnetic resonance imaging studies of older adults: a shrinking brain. $J$. Neurosci. 23, 3295-3301.

Rezin, G. T., Amboni, G., Zugno, A. I., Quevedo, J., and Streck, E. L. (2009). Mitochondrial dysfunction and psychiatric disorders. Neurochem. Res. 34, 1021-1029.

Sahin, E., and DePinho, R. A. (2012). Axis of ageing: telomeres, p53 and mitochondria. Nat. Rev. Mol. Cell Biol. 13, 397-404.

Santarelli, L., Saxe, M., Gross, C., Surget, A., Battaglia, F., Dulawa, S., et al. (2003). Requirement of hippocampal neurogenesis for the behavioral effects of antidepressants. Science 301, 805-809.

Saretzki, G. (2009). Telomerase, mitochondria and oxidative stress. Exp. Gerontol. 44, 485-492.

Schapira, A. H. (2008). Mitochondria in the aetiology and pathogenesis of Parkinson's disease. Lancet Neurol. 7, 97-109.

Sen, S., Duman, R., and Sanacora, G. (2008). Serum brain-derived neurotrophic factor, depression, and antidepressant medications: metaanalyses and implications. Biol. Psychiatry 64, 527-532.

Sharpless, N. E., and DePinho, R. A. (2004). Telomeres, stem cells, senescence, and cancer. J. Clin. Invest. 113, 160-168.

Sibille, E., Morris, H. M., Kota, R. S., and Lewis, D. A. (2011). GABA-related transcripts in the dorsolateral prefrontal cortex in mood disorders. Int. J. Neuropsychopharmacol. 14, 721-734.

Sibille, E., Su, J., Leman, S., Le Guisquet, A. M., Ibarguen-Vargas, Y. Joeyen-Waldorf, J., et al. (2007). Lack of serotonin $_{1 \mathrm{~B}}$ receptor expression leads to age-related motor dysfunction, early onset of brain molecular aging and reduced longevity. $\mathrm{Mol}$. Psychiatry 12, 1042-1056.

Smith, L. L., Coller, H. A., and Roberts, J. M. (2003). Telomerase modulates expression of growth-controlling genes and enhances cell proliferation. Nat. Cell Biol. 5, 474-479.

So, K., Tamura, G., Honda, T., Homma, N., Waki, T., Togawa, N., et al. (2006). Multiple tumor suppressor genes are increasingly methylated with age in non-neoplastic gastric epithelia. Cancer Sci. 97, 1155-1158.

Sowell, E. R., Peterson, B. S., Thompson, P. M., Welcome, S. E., Henkenius, A. L., and Toga, A. W. (2003). Mapping cortical change across the human life span. Nat.Neurosci. 6, 309-315.

Tennen, R. I., Bua, D. J., Wright, W. E., and Chua, K. F. (2011). SIRT6 is required for maintenance of telomere position effect in human cells. Nat Commun. 2, 433.

Toliver-Kinsky, T., Papaconstantinou, J., and Perez-Polo, J. R. (1997). Ageassociated alterations in hippocampal and basal forebrain nuclear factor kappa B activity. J. Neurosci. Res. 48 580-587.

Trifunovic, A., Wredenberg, A., Falkenberg, M., Spelbrink, J. N., Rovio, A. T., Bruder, C. E., et al. (2004). Premature ageing in mice expressing defective mitochondrial DNA polymerase. Nature 429, 417-423.

Tripp, A., Kota, R. S., Lewis, D. A., and Sibille, E. (2011). Reduced somatostatin in subgenual anterior cingulate cortex in major depression. Neurobiol. Dis. 42, 116-124.

Uchida, S., Hara, K., Kobayashi, A. Otsuki, K., Yamagata, H., Hobara, T., et al. (2011). Epigenetic status of Gdnf in the ventral striatum determines susceptibility and adaptation to daily stressful events. Neuron 69 359-372.

Van Orden, K., and Conwell, Y. (2011) Suicides in late life. Curr. Psychiatry Rep. 13, 234-241.

Viollet, C., Lepousez, G., Loudes, C. Videau, C., Simon, A., and Epelbaum, J. (2008). Somatostatinergic systems in brain: networks and functions. Mol. Cell. Endocrinol. 286, 75-87.

Wagner, E. F., and Nebreda, A. R. (2009). Signal integration by JNK and p38 MAPK pathways in cancer development. Nat. Rev. Cancer 9, 537-549.
Webster, M. J., Weickert, C. S., Herman, M. M., and Kleinman, J. E. (2002). BDNF mRNA expression during postnatal development, maturation and aging of the human prefrontal cortex. Dev. Brain Res. 139, 139-150.

Wolkowitz, O. M., Mellon, S. H., Epel, E. S., Lin, J., Dhabhar, F. S., Su, Y., et al. (2011a). Leukocyte telomere length in major depression: correlations with chronicity, inflammation and oxidative stress - preliminary findings. PLoS ONE 6, e17837. doi: 10.1371/journal.pone.0017837

Wolkowitz, O. M., Reus, V. I., and Mellon, S. H. (2011b). Of sound mind and body: depression, disease, and accelerated aging. Dialogues Clin. Neurosci. 13, 25-39.

Wu, X., Bishopric, N. H., Discher, D. J., Murphy, B. J., and Webster, K. A. (1996). Physical and functional sensitivity of zinc finger transcription factors to redox change. Mol. Cell. Biol. 16, 1035-1046.

Yankner, B. A., Lu, T., and Loerch, P. (2008). The aging brain. Annu. Rev. Pathol. 3, 41-66.

Zeng, Y., Tan, M., Kohyama, J., Sneddon, M., Watson, J. B., Sun, Y. E., et al. (2011). Epigenetic enhancement of BDNF signaling rescues synaptic plasticity in aging. J. Neurosci. 31, 17800-17810.

Zhou, Q. G., Hu, Y., Wu, D. L., Zhu, L. J., Chen, C., Jin, X., et al. (2011). Hippocampal telomerase is involved in the modulation of depressive behaviors. J. Neurosci. 31, 12258-12261.

Conflict of Interest Statement: The authors declare that the research was conducted in the absence of any commercial or financial relationships that could be construed as a potential conflict of interest.

Received: 31 July 2012; accepted: 16 October 2012; published online: 16 November 2012.

Citation: McKinney BC, Oh H and Sibille E (2012) Age-by-disease biological interactions: implications for late-life depression. Front. Gene. 3:237. doi: 10.3389/ fgene.2012.00237

This article was submitted to Frontiers in Genetics of Aging, a specialty of Frontiers in Genetics.

Copyright (c) 2012 McKinney, Oh and Sibille. This is an open-access article distributed under the terms of the Creative Commons Attribution License, which permits use, distribution and reproduction in other forums, provided the original authors and source are credited and subject to any copyright notices concerning any third-party graphics etc. 